and, as for the summability $(C k)$ of the series $u_{0}+u_{1}+\cdots$ $+u_{n}+\cdots$ it is necessary that*

$$
\lim _{n=\infty} \frac{u_{n}}{n^{k}}=0,
$$

it follows that, for any $k<\frac{1}{4}$, we obtain a Fourier series which is not summable $(C k)$ for any value of $x$ by selecting a $\nu$ such that $1-2 \nu>4 k$. By a suitable modification of Riemann's example, we may construct a Fourier series with the corresponding property for any $k<\frac{1}{2}$; for $1>k \geqq \frac{1}{2}$, I have not been able to decide whether the theorem is true for all integrable (and not only absolutely integrable) functions or not. $\dagger$

Chicago, Illi.,

February 3, 1913.

\title{
NOTE ON PIERPONT'S THEORY OF FUNCTIONS.
}

IN a review, written some years ago, of Pierpont's Theory of Functions of Real Variables, I made the following incorrect statement with regard to the possibility of reversing the order of differentiation of a function $f(x, y): \ddagger$

"Under the assumption that $f_{x}^{\prime}$ exists on $y=b, f_{y}^{\prime}$ on $x=a$, and that one of them is approached uniformly, it follows as a corollary to the theorem of Moore mentioned above, that the second derivatives $f_{x y}{ }^{\prime \prime}, f_{y x}{ }^{\prime \prime}$ exist at $(a, b)$ and are equal."

The assumptions should be that $f_{x}{ }^{\prime}$ exists on $x=a, f_{y}{ }^{\prime}$ on $y=b$, and that the derivative for $x$ at $x=a$ of the quotient $f(x, y) /(y-b)$ is approached uniformly for values of $y$ different from $b$. These are the hypotheses, in different words, which Professor E. H. Moore uses in the Lectures referred to on page 124 of the review, and which I intended to reproduce.

I am indebted for this correction to Mr. G. A. Pfeiffer. In a recent letter to me he cited the example $f=x y\left(x^{2}-y^{2}\right) /\left(x^{2}+y^{2}\right)$ with the agreement that $f$ shall be zero for $x=y=0$, which

* S. Chapman, 1. c., p. 379 .

$\dagger$ For $k \geqq 1$, the theorem holds for any integrable function; see for the case $k=1$ (the theorem holds a fortiori for $k>1$ ) L. Fejér, "Untersuchungen über trigonometrische Reihen," Math. Annalen, vol. 58 (1904), pp. 51-69.

$\ddagger$ BULletin, vol. 13 (1906), page 125 . 
shows that my statement was inaccurate, and he suggested also the hypotheses of Professor Moore which are given above.

G. A. Burss.

\section{REID'S THEORY OF ALGEBRAIC NUMBERS.}

The Elements of the Theory of Algebraic Numbers. By LEGH

Wilber ReId, with an Introduction by David Hilbert.

New York, The Macmillan Company, 1910. xix + 454 pp.

Professor Reid's book is the result of an attempt to present the theory of algebraic numbers through a somewhat detailed study of the numbers belonging to special quadratic realms. The first four chapters, constituting one third of the whole book, are devoted to the theory of rational integers with special emphasis upon those properties of these numbers "which find their analogues in the general theory." There can be no question but that this procedure helps the reader to appreciate the character of the generalization made through the introduction of the ideal numbers, but it is difficult to see why it is necessary to use so much space for the introduction of material easily available elsewhere, when such a course makes it necessary to omit some of the more important parts of the theory for which the book was written. The presentation of this material is good, though rather lengthy.

In the proof of the unique factorization law for rational integers much emphasis is laid upon the sequence of three well-known theorems. The first of these affirms the validity of the euclidean algorism for finding the highest common factor of two integers; the second relates to the solution in integers of the equation

$$
a x+b y=1,
$$

where $a$ and $b$ are relatively prime; while the third asserts that when the product of two integers is divisible by a prime at least one of the integers is divisible by the prime. These three theorems the author refers to as Theorems A, B, and C, respectively.

Chapters V, VI, and VII are devoted to the discussion of the integers in the realms $K(i), K(\sqrt{2})$, and $K(\sqrt{-3})$. The basis, the discriminant, the units of the realm are discussed 